\title{
Épidémie de rage du raton laveur à Hamilton, en Ontario : rapport sur l'évolution du cas
}

\author{
D Lobo ${ }^{1 *}$, C DeBenedet ${ }^{2}$, C Fehlner-Gardiner ${ }^{3}$, SA Nadin-Davis ${ }^{3}$, MEC Anderson ${ }^{4}$, T Buchanan ${ }^{5}$, \\ K Middel ${ }^{5}$, C Filejski ${ }^{6}$, J Hopkins ${ }^{7,8 *}$
}

\section{Résumé}

Contexte : La rage du raton laveur est causée par une variante du virus de la rage trouvée chez les ratons laveurs, mais transmissible aux autres espèces de mammifères, dont les humains. La maladie causée par la variante du virus de la rage du raton laveur est impossible à distinguer de la rage causée par d'autres variantes du virus.

Objectifs : Cet article décrit l'épidémie de rage du raton laveur en Ontario (découvert en décembre 2015) et les mesures de contrôle entreprises afin de freiner la propagation de l'épizootie à l'aide de l'approche Une seule santé.

Enquête et résultats : Des représentants d'organismes locaux, provinciaux et fédéraux ont activé une riposte collective à la rage du raton laveur qui comprend une mise à jour des politiques, une surveillance accrue, une campagne de sensibilisation du public et une campagne de vaccination massive de la faune sauvage et domestique. Entre les mois de décembre 2015 et de juin 2017, 338 animaux se sont révélés porteurs de la rage du raton laveur en Ontario. Bien que la majorité des cas concernait des ratons laveurs, plusieurs contagions ont touché des moufettes de même que d'autres espèces, dont deux chats, un renard et un lama. Le séquençage du génome viral a démontré que l'épizootie a probablement été causée par un transfert sur une longue distance depuis les États-Unis.

Conclusion : Cette épidémie de rage du raton laveur est de loin la plus importante à survenir au Canada et elle constitue la première à être documentée dans une zone urbaine densément peuplée. Il s'agit aussi de la première fois où cette variante du virus de la rage a été trouvée chez un animal domestique au Canada. Une approche collaborative comprenant de nombreuses parties prenantes des secteurs public et privé a été déterminante à la résolution de cette épizootie. Bien que l'incidence des cas de rage semble diminuer, plusieurs années seront probablement nécessaires avant d'atteindre une élimination complète. La poursuite de la collaboration entre ces organismes est nécessaire afin de parvenir à ce but.

Citation proposée : Lobo D, DeBenedet C, Fehlner-Gardiner C, Nadin-Davis SA, Anderson MEC, Buchanan T, Middel K, Filejski C, Hopkins J. Épidémie de rage du raton laveur à Hamilton, en Ontario : rapport sur l'évolution du cas. Relevé des maladies transmissibles au Canada. 2018;44(5):130-6. https://doi.org/10.14745/ccdr.v44i05a05f

\author{
Affiliations \\ ${ }^{1}$ Public Health and Preventive \\ Medicine Residency Program, \\ Université McMaster, Hamilton \\ (Ontario)
}

2 Hamilton Public Health Services, Hamilton (Ontario)

${ }^{3}$ Agence canadienne d'inspection des aliments, Ottawa (Ontario)

${ }^{4}$ Ministère de l'Agriculture, de I'Alimentation et des Affaires rurales de l'Ontario, Guelph (Ontario)

${ }^{5}$ Ministère des Richesses naturelles et des Forêts, Peterborough (Ontario)

${ }^{6}$ Ministère de la Santé et des Soins de longue durée, Toronto (Ontario)

${ }^{7}$ Région de Peel, Mississauga (Ontario)

${ }^{8}$ Department of Health Research Methods, Epidemiology, and Impact, Université McMaster, Hamilton (Ontario)

\section{*Correspondance : deepika. lobo@medportal.ca; jessica. hopkins@peelregion.ca}

Mots clés : Rage du raton laveur, épidémie de rage, approche Une seule santé, séquençage du génome

\section{Introduction}

Le 4 décembre 2015, le service de santé publique d'Hamilton en Ontario a été avisé qu'un raton laveur capturé dans la région était porteur de la variante du raton laveur du virus de la rage. On avait testé le raton laveur, car il s'était battu avec deux chiens domestiques non vaccinés. Ce cas de rage du raton laveur était le premier dans la province depuis 2005 et le premier cas jamais enregistré dans le sud-ouest de l'Ontario. Au cours des 19 mois suivants, des ratons laveurs porteurs de la rage ont été trouvés dans des zones autour d'Hamilton, dont la région du Niagara, le comté de Brant, les régions d'Halton et d'Haldimand-Norfolk.

Le virus de la rage est un lyssavirus de la famille des rhabdoviridae. Il possède une haute affinité pour les tissus nerveux et cause une encéphalomyélite qui entraîne la mort (1). Les variantes virales sont des populations virales qui sont maintenues dans des réservoirs hôtes particuliers dans une zone géographique donnée et qui sont distinctes des autres populations virales qui peuvent être situées dans la même région ou qui ont divergé d'un même ancêtre viral commun (2). La maladie, causée par une variante de la rage du raton laveur, est impossible à distinguer de la rage causée par d'autres variantes du virus. Par souci de concision, le terme "rage du raton laveur» employé dans le présent rapport signifie la rage causée par la variante du raton laveur du virus de la rage, que la maladie soit présente chez des ratons laveurs ou d'autres espèces.

Le présent article donne une mise à jour de l'épizootie de rage du raton laveur en Ontario, particulièrement à Hamilton, en Ontario et dans les environs où l'éclosion a été détectée en premier en décembre 2015. II décrit les mesures de contrôle 
entreprises afin de freiner la propagation de l'épizootie et I'approche collaborative Une seule santé utilisée par plusieurs organismes engagés dans la riposte.

\section{Contexte}

La rage du raton laveur a émergé à l'origine dans l'État de la Floride dans les années 1940, se propageant aux États du centre du littoral de l'Atlantique dans les années 1970. Depuis, elle s'est répandue le long de la côte est des États-Unis, atteignant la frontière canado-américaine vers le milieu des années 1990 $(3,4)$. Elle a été détectée pour la première fois en Ontario en 1995 (5) et elle a été localisée dans deux zones rurales de l'est de l'Ontario avec 132 cas détectés et confirmés en laboratoire en six ans. Elle a été éliminée avec succès en 2005 et l'Ontario a été déclaré exempte de rage du raton laveur en septembre 2007 (6). Les incursions de cas de rage du raton laveur provenant d'États américains voisins, au Nouveau-Brunswick (2000 à 2002; 64 cas) et au Québec (2006 à 2009; 104 cas), ont été éliminées de façon similaire (6).

La responsabilité du contrôle de la rage au Canada est partagée entre plusieurs paliers de gouvernement et elle est fondée sur l'approche Une seule santé, un concept qui reconnaît les liens entre la santé publique, la santé animale et l'environnement. L'approche Une seule santé agit de façon coordonnée, collaborative, multidisciplinaire et intersectorielle afin d'appréhender les risques potentiels ou existants qui originent de l'interface entre les animaux, les humains et les écosystèmes (7). Au niveau fédéral, l'Agence canadienne d'inspection des aliments (ACIA) offre des services d'épreuves de laboratoire concernant le virus de la rage pour des échantillons animaux ou humains. En Ontario, le ministère de la Santé et des Soins de longue durée oriente et soutient la gestion locale de l'exposition suspectée à la rage, les plans de contingence locaux de cas de rage et les mesures législatives entourant la vaccination antirabique. Les services de santé publique locaux sont responsables de l'ensemble des activités de prévention contre les cas de rage chez les humains, y compris la gestion des cas post-exposition de personnes potentiellement exposées au virus de la rage, de pourvoir un vaccin de prophylaxie antirabique post-exposition prophylaxie post-exposition à la rage aux fournisseurs de soins primaires et de sensibiliser le public. Le ministère de l'Agriculture, de l'Alimentation et des Affaires rurales de l'Ontario assiste au besoin les vétérinaires dans l'évaluation des risques, la soumission des échantillons et la gestion post-exposition des animaux de compagnie et du bétail (collectivement, les animaux domestiques) potentiellement exposés à la rage par les animaux sauvages ou d'autres animaux domestiques. Le ministère des Richesses naturelles et des Forêts est responsable des activités de contrôle de la rage chez la faune, ce qui comprend la surveillance accrue des populations sauvages à risque, l'analyse des échantillons et les programmes de vaccination de masse.

\section{Enquête et résultats}

\section{Coordonner une approche Une seule santé}

À la suite de la confirmation du premier cas de rage du raton laveur à Hamilton en décembre 2015, les services de santé publique d'Hamilton ont mis en place une riposte contre la rage du raton laveur fondée sur le plan de contingence contre la rage du raton laveur du ministère de la Santé et des Soins de longue durée et ils ont revu leur outil d'évaluation des risques de cas de rage afin qu'il corresponde aux changements épidémiologiques locaux (c.-à-d. un raton laveur local porteur de la rage). La mise à jour des algorithmes de gestion des cas pour une exposition humaine potentielle et la sensibilisation des fournisseurs de soins de santé locaux afin d'intégrer l'épidémiologie locale à la gestion post-exposition ont suivi.

En 2013, les services de santé publique d'Hamilton étaient I'un des six services de santé ontariens ayant participé à la mise en place d'un comité communautaire Une seule santé. Les membres du comité ont rencontré les professionnels en médecine vétérinaire locaux, les services de contrôle animal et les représentants d'autres organismes afin de discuter de la prévalence des maladies chez les humains et les animaux et de partager des renseignements portant sur les différents facteurs de risques environnementaux. Par conséquent, les services de santé publique d'Hamilton avaient créé des liens solides avant même la découverte de cette épizootie, ce qui a facilité la rapide mise en œuvre de la riposte à la rage du raton laveur.

La rage n'a pas d'impact environnemental direct, car il s'agit d'un virus de mammifère qui est très fragile à l'extérieur du corps. Par contre, elle peut avoir un impact significatif sur les populations sauvages (particulièrement sur les espèces réservoirs - les chauves-souris, les ratons laveurs, les moufettes et les renards chez lesquelles différentes variantes du virus de la rage se propagent) et de ce fait, sur les écosystèmes dans lesquels elles vivent. En ce sens, les contributions de la santé publique, du ministère de l'Agriculture, de l'Alimentation et des Affaires rurales de l'Ontario et du ministère des Richesses naturelles et des Forêts complètent les acteurs de l'initiative Une seule santé en termes de santé humaine, animale et environnementale.

Un autre élément unique de cette riposte était l'absence d'un "organisme directeur» unique; la riposte était partagée de telle sorte que chaque organisme dirigeait les aspects qui correspondaient à son mandat. Une collaboration serrée et une communication active ont été essentielles afin de coordonner les activités et le travail vers le but commun global d'éliminer la rage du raton laveur en Ontario.

\section{Réponse en santé animale}

\section{Définition du cas}

Un cas confirmé de rage du raton laveur était défini comme un animal déclaré porteur du virus de la rage à la suite d'une analyse en laboratoire réalisée à l'aide d'une épreuve d'immunofluorescence et d'une méthode d'identification des virus employant soit des panels d'anticorps monoclonaux ou le séquençage afin de déterminer la variante raton laveur du virus. Ces procédures ont été effectuées par le Centre d'expertise de la rage de l'Agence canadienne d'inspection des aliments. L'attribution géographique des cas positifs était fondée sur les frontières des services de santé publique locaux.

\section{Détection des cas et surveillance}

Les cas ont été identifiés par différents moyens selon que les personnes ou les animaux domestiques avaient été potentiellement exposés à l'animal suspecté ou que l'animal suspecté avait été ciblé par d'autres activités de surveillance. La décision de procéder à une analyse d'un animal suspecté qui 
avait potentiellement exposé des humains à la rage était prise par le service de santé publique local. La décision de procéder à une analyse d'un animal suspecté qui avait potentiellement exposé des animaux domestiques seuls à la rage était prise par le ministère de l'Agriculture, de l'Alimentation et des Affaires rurales de l'Ontario, d'ordinaire en collaboration avec le vétérinaire de l'animal exposé. Dans les deux cas, les échantillons étaient envoyés directement à l'Agence canadienne d'inspection des aliments à des fins d'analyse.

En décembre 2015, le ministère des Richesses naturelles et des Forêts a accru la surveillance des cas de rage dans une zone s'étendant à $50 \mathrm{~km}$ au-delà de l'endroit où tous les cas diagnostiqués ont été trouvés, avec des analyses hebdomadaires des animaux malades ou trouvés morts sans aucun contact connu avec des humains ou des animaux domestiques, en pratiquant une épreuve immunohistochimique directe rapide (8). Des épreuves de contrôle sur l'ensemble des échantillons d'épreuves immunohistochimiques directes rapides positives et inconcluantes ont été effectuées par l'Agence canadienne $d^{\prime}$ inspection des aliments. Cette surveillance accrue a servi à comprendre l'étendue (espèces et géographie) de la propagation et le nombre d'animaux affectés.

Entre les mois de décembre 2015 et juin 2017, 338 cas se sont révélés porteurs de la rage du raton laveur en Ontario. De ceux-ci, 251 cas (74 \%) provenaient des environs d'Hamilton. Au moins cinq animaux se révélaient positifs à la rage du raton laveur chaque mois, de décembre 2015 à juin 2017, avec une moyenne de 18 animaux par mois (écart de 5 à 30). En revanche, en 2014, seulement 18 cas de rage avaient été détectés dans I'ensemble de la province, tous chez les chauves-souris (9). Une courbe épizootique de l'épidémie est montrée à la figure 1.

Figure 1 : Courbe épizootique montrant le déroulement de l'épidémie de rage du raton laveur en Ontario, de décembre 2015 à juin 2017

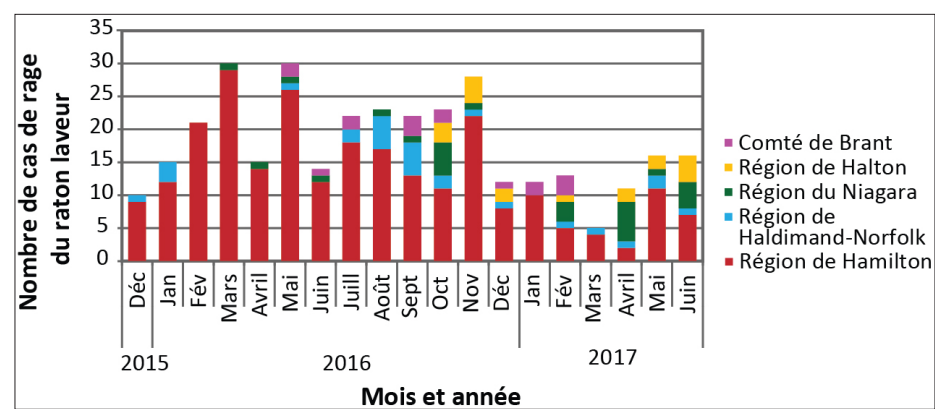

Alors que l'épidémie de rage était surtout confinée aux ratons laveurs, I'espèce réservoir de cette variante du virus, un nombre significatif de transmissions interespèces du virus du raton laveur (contagion) ont été observés chez la moufette de même que quelques contagions vers d'autres espèces, dont deux chats, un renard roux et un lama (tableau 1 ).

Entre décembre 2015 et juin 2017, le ministère des Richesses naturelles et des Forêts a procédé à des analyses en laboratoire sur 6685 animaux (espèces sauvages sans aucune exposition connue aux humains ou aux animaux domestiques) à l'aide d'épreuves d'immunohistochimie directe rapide. De ceux-ci, 326 cas $(4,89 \%)$ ont été identifiés comme porteurs de la rage du raton laveur à l'aide d'épreuves d'immunohistochimie directe rapide et confirmés par des épreuves d'immunofluorescence et d'identification de la variante du virus par la suite (tableau 2).
Tableau 1 : Espèces animales chez lesquelles les analyses en laboratoire ont révélé la présence de rage du raton laveur en Ontario, décembre 2015 à juin 2017

\begin{tabular}{|l|l|r|r|r|r|r|r|}
\hline \multirow{2}{*}{ Année } & Animal & \multicolumn{1}{|c|}{$\begin{array}{c}\text { Région } \\
\text { de } \\
\text { Hamilton }\end{array}$} & $\begin{array}{c}\text { Région de } \\
\text { Haldimand- } \\
\text { Norfolk }\end{array}$ & $\begin{array}{c}\text { Région } \\
\text { du } \\
\text { Niagara }\end{array}$ & $\begin{array}{c}\text { Région } \\
\text { d'Halton }\end{array}$ & $\begin{array}{c}\text { Comté } \\
\text { de } \\
\text { Brant }\end{array}$ & TOTAL \\
\hline $\begin{array}{l}2015 \\
\text { (Décembre) }\end{array}$ & $\begin{array}{l}\text { Raton } \\
\text { Laveur }\end{array}$ & 9 & 1 & 0 & 0 & 0 & 10 \\
\hline \multirow{3}{*}{2016} & $\begin{array}{l}\text { Raton } \\
\text { laveur }\end{array}$ & 126 & 17 & 10 & 7 & 11 & 171 \\
\cline { 2 - 9 } & Moufette & 76 & 1 & 2 & 2 & 0 & 81 \\
\cline { 2 - 9 } & Chat & 0 & 1 & 0 & 0 & 0 & 1 \\
\cline { 2 - 9 } & Renard & 1 & 0 & 0 & 0 & 0 & 1 \\
\cline { 2 - 9 } & Lama & 0 & 1 & 0 & 0 & 0 & 1 \\
\hline \multirow{2}{*}{$\begin{array}{l}\text { (Janvier à } \\
\text { juin) }\end{array}$} & $\begin{array}{l}\text { Raton } \\
\text { laveur }\end{array}$ & 24 & 5 & 12 & 4 & 5 & 50 \\
\cline { 2 - 9 } & Moufette & 14 & 1 & 2 & 5 & 0 & 22 \\
\cline { 2 - 9 } & Chat & 1 & 0 & 0 & 0 & 0 & 1 \\
\hline \multirow{2}{*}{ TOTAL } & & 251 & 27 & 26 & 18 & 16 & 338 \\
\hline
\end{tabular}

Douze cas additionnels ont été découverts pendant ce temps à l'aide d'échantillons soumis par le ministère de la Santé et des Soins de longue durée et le ministère de l'Agriculture, de I'Alimentation et des Affaires rurales de l'Ontario.

Tableau 2 : Nombre d'animaux sur lesquels des analyses en laboratoire ont été effectuées à l'aide d'épreuves d'immunohistochimie directe rapide afin de déceler la rage en Ontario, décembre 2015 à juin 2017

\begin{tabular}{|c|c|c|c|c|}
\hline Année & Mois & $\begin{array}{c}\text { Nombre } \\
\text { d'animaux } \\
\text { analysés }\end{array}$ & $\begin{array}{c}\text { Nombre } \\
\text { d'animaux } \\
\text { confirmés } \\
\text { comme étant } \\
\text { porteurs }\end{array}$ & $\begin{array}{l}\text { Proportion } \\
\text { des animaux } \\
\text { confirmés } \\
\text { comme étant } \\
\text { porteurs (\%) }\end{array}$ \\
\hline 2015 & Déc & 147 & 12 & 8.2 \\
\hline \multirow[t]{12}{*}{2016} & Jan & 369 & 19 & 5.2 \\
\hline & Fév & 588 & 26 & 4.4 \\
\hline & Mars & 781 & 19 & 2.4 \\
\hline & Avril & 627 & 23 & 3.7 \\
\hline & Mai & 453 & 24 & 5.3 \\
\hline & Juin & 338 & 16 & 4.7 \\
\hline & Juil & 274 & 18 & 6.6 \\
\hline & Août & 304 & 23 & 7.6 \\
\hline & Sept & 228 & 27 & 11.8 \\
\hline & Oct & 247 & 22 & 8.9 \\
\hline & Nov & 255 & 19 & 7.5 \\
\hline & Déc & 123 & 11 & 8.9 \\
\hline \multirow[t]{6}{*}{2017} & Jan & 121 & 12 & 9.9 \\
\hline & Fév & 277 & 13 & 4.7 \\
\hline & Mars & 310 & 3 & 1.0 \\
\hline & Avril & 377 & 12 & 3.2 \\
\hline & Mai & 445 & 16 & 3.6 \\
\hline & Juin & 421 & 11 & 2.6 \\
\hline Total & --- & 6,685 & 326 & 4.9 \\
\hline
\end{tabular}




\section{Identification de la source}

La source de cette épidémie a été explorée en comparant la séquence de l'ensemble du génome de deux isolats du virus issus de Hamilton à l'aide d'une grande base de données de génomes de différentes variantes du virus de la rage du raton laveur provenant de partout dans l'est des États-Unis. Les virus actifs tout juste de l'autre côté de la frontière avec l'État de New York, où la rage du raton laveur est endémique depuis la fin des années 1990, étaient philogénétiquement très différents de ceux qui étaient responsables de l'épidémie d'Hamilton, soutenant la conclusion que cette épizootie représente un transfert à longue distance dans la région (10).

\section{Vaccination}

La vaccination des animaux était l'une des principales stratégies employées afin de minimiser la propagation de la rage du raton laveur, particulièrement au moyen de la vaccination orale des ratons laveurs et des moufettes. Entre décembre 2015 et juin 2017, le ministère des Richesses naturelles et des Forêts a distribué plus de 1,7 million d'appâts contenant le vaccin oral antirabique $O N R A B^{\circledR}$ (vecteur adénoviral vivant AdRG 1.3) au cours des campagnes de décembre 2015, du printemps 2016 et de l'automne 2016. Les appâts ont été distribués à la main dans les zones urbaines, par hélicoptère dans les grands espaces verts urbains et par aéronef à voilure fixe dans les zones rurales environnantes afin d'accroître la zone de surveillance.

De plus, les services de santé publique d'Hamilton, conjointement avec les vétérinaires locaux, ont tenu deux cliniques de vaccination antirabique pour les chats et les chiens domestiques en septembre 2016 et en avril 2017. Ces cliniques ont été organisées afin d'offrir la vaccination antirabique à coût moins élevé, ce qui a permis aux familles à faible revenu de faire vacciner leurs animaux domestiques. Treize vétérinaires y ont participé, en vaccinant 472 animaux domestiques au total (321 chiens et 151 chats). De ceux-ci, 169 animaux (36\%) n'avaient aucun antécédent de vaccination. Les services de santé publique d'Hamilton poursuivent leur travail auprès de Community Veterinary Outreach, un organisme qui fournit des soins aux animaux domestiques dont les propriétaires sont des personnes à faibles revenus. Le but est d'offrir la vaccination antirabique à un coût abordable pour que les animaux domestiques et les humains soient protégés de la rage.

\section{Riposte des services de santé publique}

\section{Formation et sensibilisation}

Les services de santé publique d'Hamilton ont élaboré une campagne de sensibilisation nommée «Rabies is Real» (La rage est réelle), exigeant que les personnes ne s'approchent pas des animaux sauvages, qu'elles signalent à leurs services animaliers les animaux décédés ou qui présentent un comportement anormal, qu'elles communiquent avec les services de santé publique si elles sont mordues ou égratignées par un animal et qu'elles fassent vacciner leurs animaux de compagnie. Le matériel de la campagne comprenait des affiches pour panneaux d'affichage, des cahiers à colorier destinés aux enfants, des messages à publier sur les réseaux sociaux, des articles de journaux, des entrevues à la télévision, une vidéo de sensibilisation à la rage et des sessions d'information destinées aux groupes à haut risque (p. ex. les groupes de bien-être des animaux). Des estimations du nombre de personnes rejointes par les panneaux d'affichage ont été fournies par leurs propriétaires et la visibilité des réseaux sociaux a été calculée par l'équipe des communications.

La campagne «Rabies is Real» a été lancée en septembre 2016. On a estimé que les dix-sept panneaux d'affichage situés à de multiples endroits dans Hamilton ont été vus plus de huit millions de fois au cours d'une période de douze semaines. On a estimé que les enseignes de la campagne placées dans 46 abribus auraient été vues plus de treize millions de fois au cours d'une période de huit semaines. La page Web de la ville de Hamilton a attiré 7393 visiteurs différents entre le mois d'octobre 2016 et le mois de mai 2017, chaque visiteur passant en moyenne 3,6 minutes sur la page Web et $83 \%$ de ceux-ci quittant la page sans aller ailleurs sur le site Web.

\section{Prophylaxie antirabique post-exposition (PAPE)}

Les services de santé publique d'Hamilton ont envoyé des conseillers médicaux auprès des fournisseurs de soins primaires afin d'informer ces derniers de l'épidémie de rage du raton laveur et des critères régissant la prophylaxie antirabique post-exposition (PAPE). Ils ont aussi créé une vidéo éducative sur la prestation de la PAPE. En comparaison avec l'année 2015, on a observé une augmentation de $52 \%$ du nombre de personnes ayant reçu la prophylaxie post-exposition à la rage dans la région de Hamilton en 2016 (figure 2).

Figure 2 : Nombre de clients ayant reçu la prophylaxie post-exposition à la rage dans la région de Hamilton en 2015 et 2016.

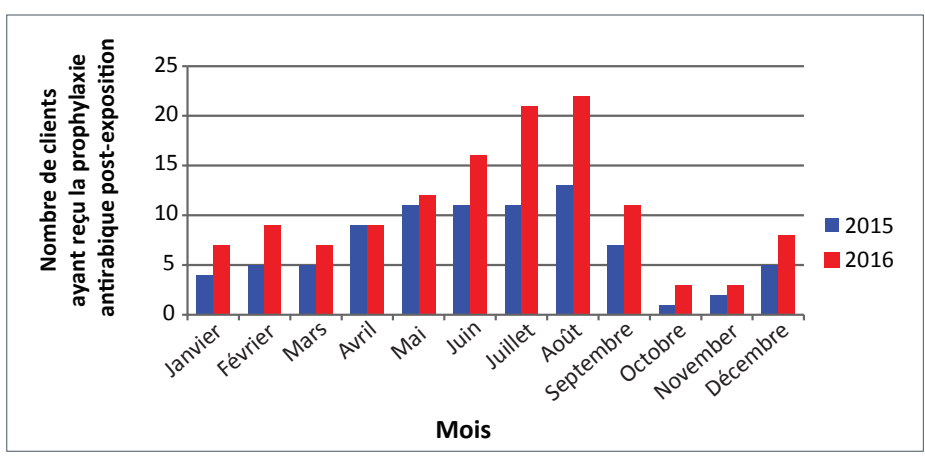

L'approche Une seule santé à l'épidémie de rage du raton laveur en Ontario est résumée au tableau 3. 
Tableau 3 : résumé de l'approche Une seule santé à l'épidémie de rage du raton laveur, Ontario 2015 à 2017

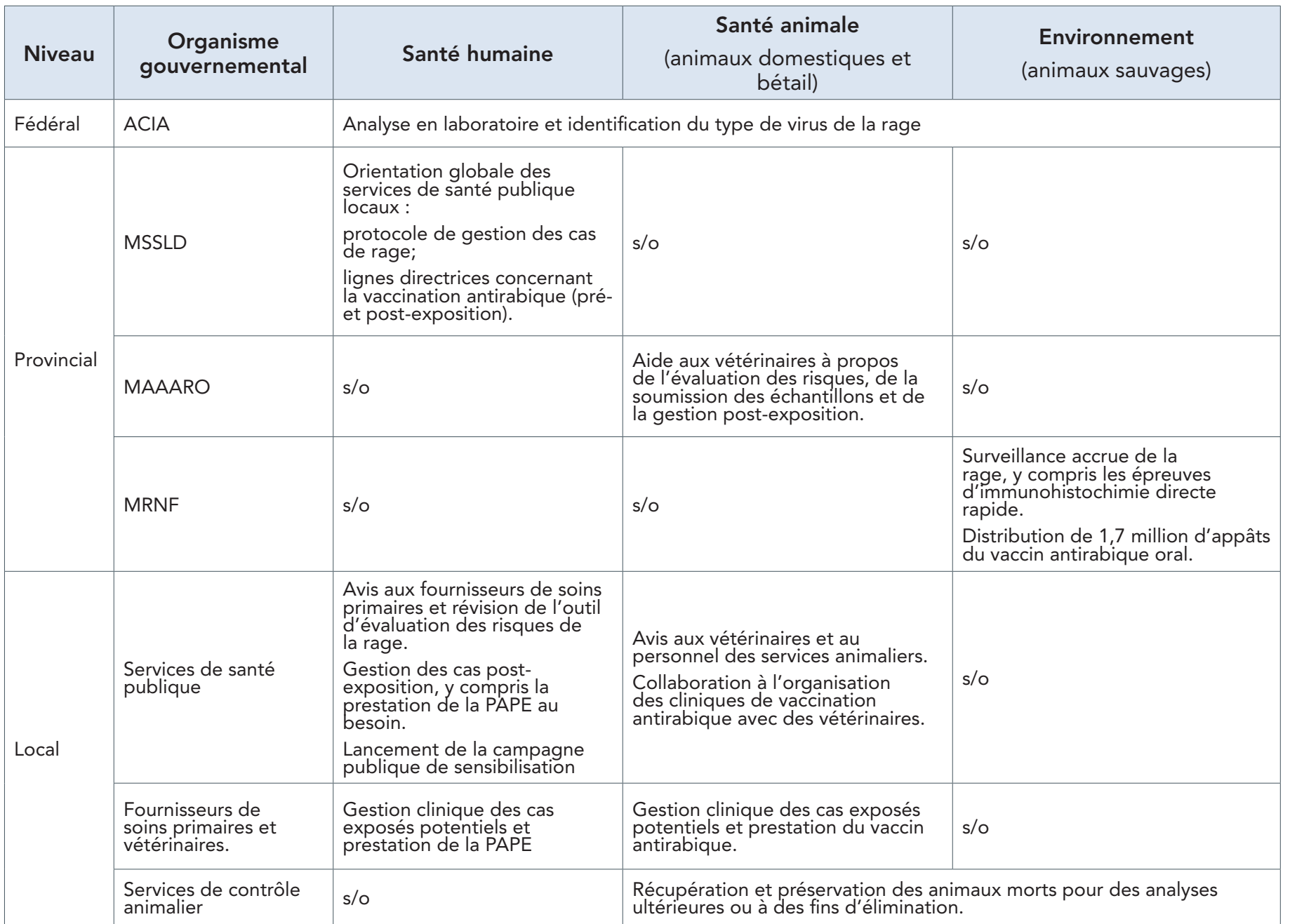

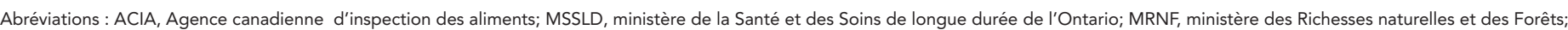
$\mathrm{s} / \mathrm{o}$, sans objet; MAAARO, ministère de l'Agriculture, de I'Alimentation et des Affaires rurales de I'Ontario; PAPE, prophylaxie antirabique post-exposition

\section{Discussion}

Au moment de la publication, la région de Hamilton et ses environs sont toujours aux prises avec une épizootie de rage du raton laveur. L'épidémie de rage du raton laveur qui sévit dans le sud-ouest de l'Ontario est de loin la plus importante à être survenue au Canada. Elle est aussi différente des épidémies précédentes qui ont eu lieu en Ontario, au Québec et au Nouveau-Brunswick en ce sens qu'elle est située dans une zone urbaine densément peuplée. Le contrôle de cette épidémie présente plusieurs défis. Par exemple, la distribution d'appâts par des vols à basse altitude d'aéronef à voilure fixe, comme c'est la norme pour les campagnes de vaccination orale en milieu rural, n'est pas possible dans les milieux urbains. Cette épidémie a nécessité une combinaison d'approches pour la distribution de vaccins antirabiques oraux, comme la distribution d'appâts à la main dans les centres urbains et par hélicoptères dans les grands espaces verts urbains. La distribution d'appâts dans les centres urbains nécessite la coordination de la messagerie entre les services de santé publique d'Hamilton, du ministère des Richesses naturelles et des Forêts et du ministère de l'Agriculture, de l'Alimentation et des Affaires rurales de l'Ontario afin d'aviser le public qui croiserait ou entrerait en contact avec ces appâts.
Une complication additionnelle de cette épidémie de rage en zone urbaine est les importantes populations d'hôtes potentiels de la contagion, comme les chats et les chiens, qui augmentent d'autant la préoccupation du risque accru de transmission aux humains. En effet, même si la majorité des cas ont été observés chez les animaux sauvages, c'est la première fois que la variante du raton laveur du virus de la rage est observée chez les espèces animales domestiques au Canada. Aux États-Unis, l'espèce animale domestique chez laquelle les cas de rage sont le plus souvent signalés est le chat, avec une majorité des cas provenant de régions où la rage du raton laveur est endémique (11). À mesure que l'épidémie progresse, le risque s'accroît que des cas de rage du raton laveur surviennent chez des espèces animales domestiques. Face à cette situation, les messages clés de la santé publique demandent d'encourager la vaccination de tous les chiens et les chats et d'éviter les contacts avec des animaux errants dont il est peu probable qu'ils aient été vaccinés. Ceci représente un réel défi, car plusieurs personnes décident de ne pas faire vacciner leurs animaux domestiques malgré l'exigence légale existant en Ontario.

Au Canada, la surveillance de la rage est fondée sur un modèle passif où seuls les animaux que l'on suspecte d'avoir potentiellement été en contact avec des personnes ou des 
animaux domestiques sont soumis à un test de dépistage de la rage. Depuis la détection de l'épidémie en décembre 2015, la vaste majorité des cas (plus de $96 \%$ ) ont été détectés au moyen du programme de surveillance accrue de la rage mis en œuvre par le ministère des Richesses naturelles et des Forêts. Une telle surveillance contribue à délimiter l'étendue de l'épidémie, ce qui éclaire les décisions de gestion en ce qui a trait aux zones de vaccination orale de même qu'à la détermination des risques pour la santé publique. Ces données renseignent aussi sur I'utilité d'aller au-delà du modèle de surveillance passive afin d'intégrer le dépistage des animaux trouvés morts ou de ceux démontrant des symptômes cliniques compatibles avec la rage, quels que soient leurs antécédents d'exposition aux humains ou aux animaux domestiques. De telles actions peuvent contribuer à une détection précoce des nouvelles éclosions de rage. Cependant, le coût d'un tel programme, particulièrement en l'absence de preuve d'une éclosion de la maladie, doit être examiné le plus sérieusement possible. II est important de trouver un équilibre entre le risque qu'une éclosion demeure non détectée pendant un certain temps et le coût des programmes de surveillance accrue.

Par le passé, l'introduction de la rage du raton laveur au Canada a typiquement été le résultat d'une propagation transfrontalière et de ce fait, les variantes virales étaient très similaires de part et d'autre de la frontière canado-américaine. Cependant, I'analyse génétique du virus en circulation à Hamilton démontre qu'une simple propagation transfrontalière n'était pas la source de l'épidémie en cours. Il apparaît que la maladie a été introduite par un transfert à longue distance d'un raton malade, soit par la voie maritime (navigation) ou par la voie terrestre (transport par camion), ce qui a été décrit auparavant (12). Ainsi, même les instances où le risque d'éclosion de rage est jugé faible devraient mettre en place des plans de contingence au cas où un tel événement se produirait. L'existence préalable de plans de contingence au ministère de la Santé et des Soins de longue durée de l'Ontario (mesures pour la santé humaine) et au ministère des Richesses naturelles et des Forêts (contrôle de la rage chez la faune sauvage) de même que l'existence du comité communautaire Une seule santé à Hamilton ont été des facteurs qui ont contribué à la rapidité avec laquelle les mesures de riposte impliquant différents programmes d'actions ont été mises en place.

Les épizooties de rage du raton laveur précédentes ont été éliminées des provinces de l'Ontario, du Québec et du Nouveau-Brunswick en six, quatre et trois ans, respectivement. Avec la taille de l'épizootie en cours, et le défi particulier de gérer une épidémie en milieu urbain, il n'est pas surprenant de constater que cette épidémie perdure depuis plus de 19 mois. Cependant, la diminution du nombre de cas observés par mois et du pourcentage d'animaux qui se sont révélés porteurs de la rage à la suite d'analyses en laboratoire suggère que l'intensité de l'épizootie pourrait être en train de décroître. L'approche collaborative entre les différents organismes interdisciplinaires aux niveaux fédéral (ACIA), provincial (MSSLD, MAAARO et le ministère des Richesses naturelles et des Forêts) et local (services de santé publique, services de contrôle animalier locaux et vétérinaires privés) a été déterminante à la résolution de la présente épizootie de rage du raton laveur. La poursuite de cette collaboration est nécessaire afin d'éliminer une fois de plus la rage du raton laveur en Ontario.

\section{Déclaration des auteurs}

D. L. - Conceptualisation, analyse formelle, rédaction de la première ébauche, examen et révision, visualisation C. D. B. - Rédaction - examen et révision, enquête

C. F. G. - Rédaction - examen et révision, enquête

S. A. N. D. - Rédaction - examen et révision, enquête

M. E. C. A. - Rédaction - examen et révision

T. B. - Rédaction - examen et révision, analyse formelle, enquête

K. M. - Rédaction - examen et révision, enquête

C. F. - Rédaction - examen et révision

J. H. - Conceptualisation, rédaction - examen et révision, enquête, supervision

\section{Conflit d'intérêt}

Aucun.

\section{Financement}

Aucun.

\section{Remerciements}

Nous remercions les membres du personnel au services de santé publique de Hamilton, au ministère de la Santé et des Soins de longue durée de l'Ontario, au ministère de l'Agriculture, de l'Alimentation et des Affaires rurales de l'Ontario et au Programme de surveillance de la faune et de recherche du ministère des Richesses naturelles et des Forêts ainsi qu'au laboratoire de diagnostic de la rage de l'Agence canadienne d'inspection des aliments à Ottawa qui ont contribué aux actions de riposte contre la rage.

\section{Références}

1. Wunner WH, Conzelmann KK. Chapter 2: Rabies virus. In: Jackson AC, editor. Rabies - Scientific basis of the disease and its management. 3rd ed. San Diego: Elsevier Inc; 2013. pp. 17-60.

2. Nadin-Davis SA. Chapter 4: Molecular Epidemiology. In: Jackson AC, editor. Rabies - Scientific basis of the disease and its management. 3rd Edition). San Diego: Elsevier Inc; 2013. pp. 123-77.

3. Hanlon CA, Childs JE. Chapter 3: Epidemiology. In: Jackson AC, editor. Rabies - Scientific basis of the disease and its management. 3rd ed. San Diego: Elsevier Inc; 2013. pp. 61121.

4. Centers for Disease Control and Prevention (CDC). Update: raccoon rabies epizootic--United States and Canada, 1999. MMWR Morb Mortal Wkly Rep 2000 Jan;49(2):31-5. PubMed (https://www.ncbi.nlm.nih.gov/ pubmed/10680602?dopt=Abstract)

5. Wandeler Al, Salsberg EB. ONTARIO. Raccoon rabies in eastern Ontario. Can Vet J 1999 Oct;40(10):731. PubMed (https://www.ncbi.nlm.nih.gov/ pubmed/17424571?dopt=Abstract)

6. Stevenson B, Goltz J, Massé A. Préparation et intervention suite aux cas récents de rage du raton laveur au 
Canada. Relevé des maladies transmissibles au Canada. 2016;42(6):140-4. http://dx.doi.org/10.14745/ccdr.v42i06a03

7. One Health Global Network. What is One Health? http://www. onehealthglobal.net/what-is-one-health/

8. Middel K, Fehlner-Gardiner C, Pulham N, Buchanan T. Incorporating direct rapid immunohistochemical testing into large-scale wildlife rabies surveillance. TropicalMed 2017;2(3):21. http://dx.doi.org/10.3390/tropicalmed2030021

9. Agence canadienne d'inspection des aliments. Cas de rage au Canada 2014 [Consulté le 14 oct 2017]. http://www. inspection.gc.ca/animaux/animaux-terrestres/maladies/ declaration-obligatoire/rage/cas-de-rage-au-canada-2014/ $\mathrm{fra} / 1424879193262 / 1424879195231$

10. Trewby H, Nadin-Davis SA, Real LA, Biek R. Processes underlying rabies virus incursions across US-Canada border as revealed by whole-genome phylogeography. Emerg Infect Dis 2017 Sep;23(9):1454-61. http://dx.doi.org/10.3201/ eid2309.170325. PubMed (https://www.ncbi.nlm.nih.gov/ pubmed/28820138?dopt=Abstract)

11. Birhane MG, Cleaton JM, Monroe BP, Wadhwa A, Orciari LA, Yager P, Blanton J, Velasco-Villa A, Petersen BW, Wallace RM. Rabies surveillance in the United States during 2015. J Am Vet Med Assoc 2017 May;250(10):1117-30. http://dx.doi. org/10.2460/javma.250.10.1117. PubMed (https://www.ncbi. nlm.nih.gov/pubmed/28467751?dopt=Abstract)

12. Smith DL, Waller LA, Russell CA, Childs JE, Real LA. Assessing the role of long-distance translocation and spatial heterogeneity in the raccoon rabies epidemic in Connecticut. Prev Vet Med 2005 Oct;71(3-4):225-40. http://dx.doi. org/10.1016/j.prevetmed.2005.07.009. PubMed (https:// www.ncbi.nlm.nih.gov/pubmed/16153724?dopt=Abstract)

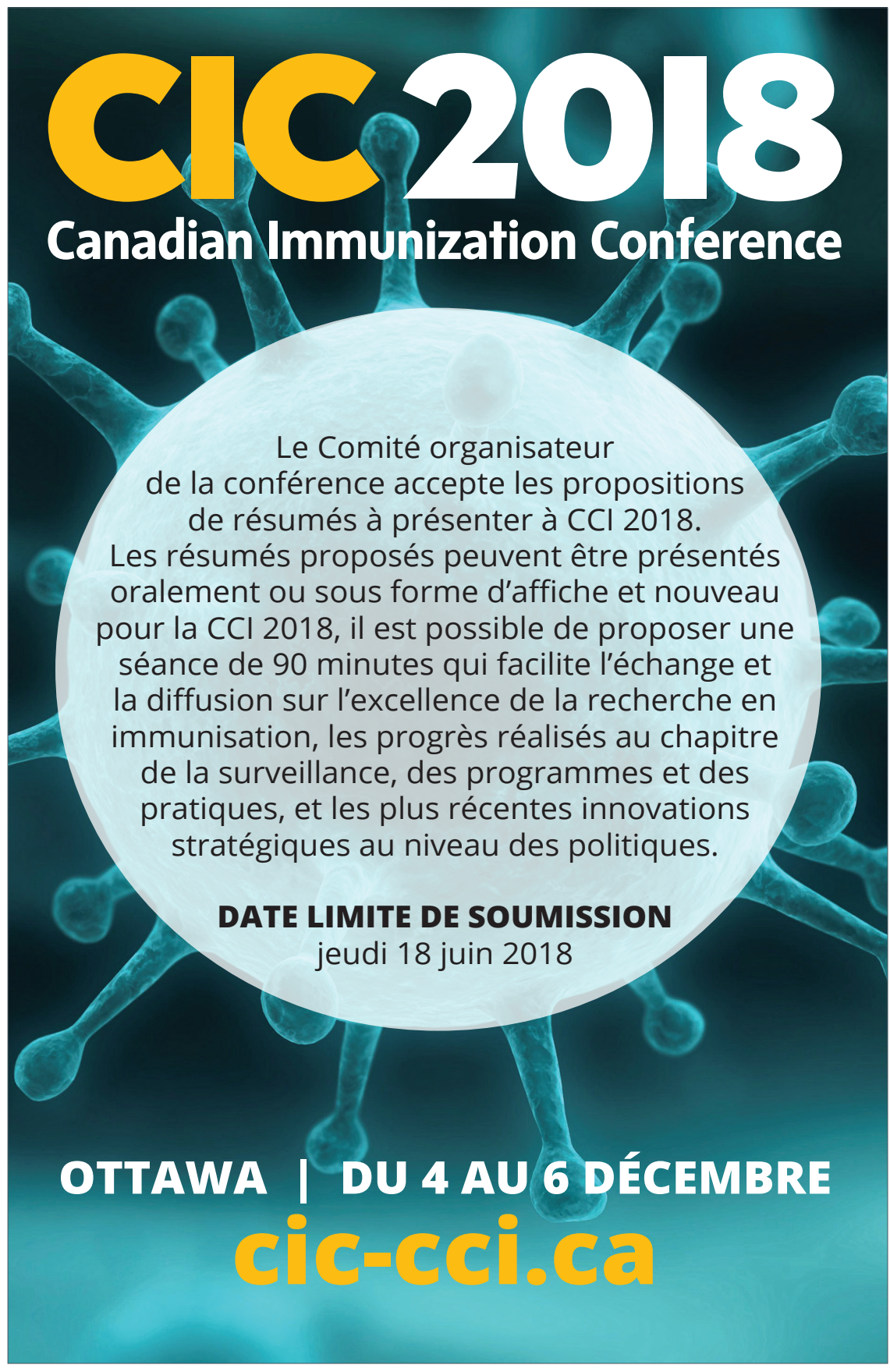

